\title{
Type-2 Diabetes Mellitus and Oral Tumors in Hungary: A Long-term Comparative Epidemiological Study
}

\author{
DÁNIEL VÉGH ${ }^{1}$, DOROTTYA BÁNYAI ${ }^{2}$, PÉTER HERMANN $^{1}$, ZSOLT NÉMETH $^{3}$ and MÁRTA UJPÁL ${ }^{3}$ \\ Departments of ${ }^{1}$ Prosthodontics, ${ }^{2}$ Pedodontics and Orthodontics and \\ ${ }^{3}$ Oral and Maxillofacial Surgery, Semmelweis University, Budapest, Hungary
}

\begin{abstract}
Background: Hungary has a leading place in global statistics of oral cancer and there is a high incidence of type-2 diabetes. Patients and Methods: A retrospective diabetes and impaired fasting glucose (IFG) screening of 758 inpatients with histologically-confirmed oral malignancies was performed. The control group comprised of 534 tumorfree adults. The results were compared to data collected 14 years earlier. Results: Diabetes mellitus (DM) was found in $25.9 \%$ and IFG in $20.6 \%$ of patients with oral cancer. Fourteen years earlier, DM was diagnosed in $14.6 \%$ and IFG in $9.7 \%$ of cancer patients. Among diabetic patients, the labial tumor location showed long-term dominance. The smoking rate of diabetes patients with oral cancer was higher in the present than in the previous study. Conclusion: The prevalence of type 2 diabetes and IFG increased significantly among oral cancer patients in the last 14 years.
\end{abstract}

Diabetes is considered an endemic medical condition with increasing prevalence globally. This tendency has been also detected in Hungary where the prevalence of diabetes among the adult population reached $7 \%$ according to the latest statistical data (1). Numerous data from several medical publications have shown that diabetes is a promoting factor in the formation of malignant tumors at different sites and has a negative impact on tumor progression and patient survival (2-16). Our medical research team has long studied the correlations between oral cavity cancer and diabetes, mainly type 2 diabetes $(17,18)$. Since type-2 diabetes has rapidly increased in prevalence globally and the morbidity and mortality rates of oral cavity cancer -especially in

This article is freely accessible online.

Correspondence to: Dr. Dániel Végh, Faculty of Dentistry, Department of Prosthodontics, Semmelweis University, H-1088 Budapest, Szentkirályi utca 47, Hungary. Tel: +36 13384380, e-mail: vegh.daniel@dent.semmelweis-univ.hu

Key Words: Type-2 diabetes, oral cancer, IFG, smoking, risk factor.
Hungary- do not show any significant decrease, we examined the relationship between glucose metabolic disorders and oral cancer in our clinic over the past 14 years. In the present study, we analyzed data of 758 patients with oral cavity cancer and 534 tumor-free control patients and examined the prevalence of diabetes and impaired fasting glucose (IFG), the distribution of tumor location and the role of smoking. Additionally, we compared our current results to data obtained 14 years ago.

\section{Patients and Methods}

We conducted a retrospective research study in the inpatient ward of the Semmelweis University Department of Oral and Maxillofacial Surgery, Budapest, Hungary. We examined patients who were diagnosed with histologically confirmed malignant oral tumors. The examination period was between the 1st of January, 2012 and 31st of December, 2015. These results were compared to an earlier study that was conducted in the same Department between the 1st of January, 1998 and 30th of June, 2002.

We processed the data of 610 patients (435 males and 175 females) in the first interval and the data of 758 patients (400 male and 358 female) in the second interval. All patients were diagnosed with histologically confirmed malignant cancer. Based on these histological data, we confirmed that 606 cases among the 610 and 749 cases among the 758 were squamous cell carcinomas, while the remainder consisted of adenocarcinomas. The average age of the patients from the previous study was 56 years (range $=36-85$ ), whereas the average age of the patients in the follow-up study was 64 years (range $=12-92$ ).

The control group in the first research interval was formed by 574 patients ( 351 male, 223 female). Their average age was 51 years (range $=32-79$ ).

The control group in the second study interval was formed by 534 patients ( 318 male, 216 female) with an average age of 53 years (range=30-94). The patients of the control group in both study intervals volunteered for stomato-oncological screening and found symptom- and cancer-free.

We considered patients with diabetes mellitus (DM) to be those who had been previously diagnosed and treated for diabetes by an internist or diabetologist. We classified patients into the IFG group when their fasting blood glucose values were between 6.1 and 6.9 $\mathrm{mmol} / \mathrm{l}$. We considered patients to have normal glucose metabolism when their blood sugar levels were below $6.1 \mathrm{mmol} / 1$. 
The laboratory test was conducted in the Central Laboratory of Semmelweis University using a Beckman Coulter AU 5800 laboratory diagnostic machine (Beckman Coulter Inc., Brea, CA, USA).

We compared the prevalent locations of the malignant oral tumors and the smoking habits between the group with diabetes and the group without diabetes.

We used Pearson's Chi-squared test $\left(\chi^{2}\right)$ for our statistical analysis. Differences below a 5\% limit $(p<0.05)$ were considered significant.

\section{Results}

DM was found in $25.9 \%$ (196 patients) and IFG in $20.6 \%$ (156 patients) of the oral cancer group. In the control group, the rate of DM was $10.3 \%$ (55 patients), whereas the rate of IFG was $10.5 \%$ (56 patients). This difference was significant among both the DM group and the IFG group of patients $(p<0.05)$. In the diabetes mellitus group, $98 \%$ were diagnosed with type 2 diabetes (192 patients), whereas the remaining $2 \%$ had insulin-treated type 1 diabetes.

These results indicated that $46.5 \%$ of the oral cancer group had a metabolic disorder (IFG + DM). In the tumorfree control group of 534 patients, $10.3 \%$ (55 patients) had DM and $10.5 \%$ (56 patients) IFG. In the control group, $20.8 \%$ (111) had a glucose metabolic disorder. The rate of metabolic disorder in the oral cancer group was significantly higher than that in the control group $(p<0.05)$.

Upon comparing the present study to the data that we collected 14 years earlier, we found that the rate of diabetes increased from $14.6 \%$ to $25.9 \%$, while the rate of IFG increased from $9.7 \%$ to $20.6 \%(p<0.05)$. Among the tumorfree control group, the rate of DM climbed from $5.6 \%$ to $10.3 \%$, as did IFG from $5.5 \%$ to $10.5 \%$, which was statistically significant $(p<0.05)$ (Table I).

Based on the statistical analysis, we concluded that the prevalence of patients with glucose metabolic disorders (IFG + DM) markedly increased from the previously measured $24.3 \%$ to $46.5 \%$ among those patients with cancer. We also found that the number of patients with glucose metabolic disorders (IFG + DM) climbed from $11.1 \%$ to $20.8 \%$ over time among the control group, which was statistically significant $(p<0.05)$.

The most common cancer locations in the non-diabetes oral cancer group were gingival cancer (30.2\%) and sublingual malignant tumors $(22.1 \%)$. The most frequent types of tumors among patients with diabetes were labial and lingual cancers $(34.7 \%$ and $21.4 \%$, respectively) (Table II).

The frequency of smoking among oral cancer patients, cancer patients with diabetes and control group patients was $57.7 \%, 64.3 \%$ and $41.2 \%$, respectively. A previous study reported smoking rates of $68 \%, 30 \%$ and $28 \%$ among oral cancer patients, cancer patients with diabetes and control group patients, respectively (Table III).
Table I. Prevalence of IFG and diabetes among patients diagnosed with oral cancer and the control group at two different examination intervals.

\begin{tabular}{lcclll}
\hline & \multicolumn{2}{c}{ Oral cancer group } & & \multicolumn{2}{c}{ Control group } \\
\cline { 2 - 3 } \cline { 5 - 6 } & $1998-2002$ & $2012-2015$ & & $1998-2002$ & $2012-2015$ \\
\cline { 5 - 6 } & $14.6 \%$ & $25.9 \%$ & & $5.6 \%$ & $10.3 \%$ \\
DM & $9.7 \%$ & $20.6 \%$ & & $5.5 \%$ & $10.5 \%$ \\
\hline
\end{tabular}

DM, Diabetes mellitus, IFG, impaired fasting glucose.

Our results led us to conclude that the rate of smoking increased among oral cancer patients with diabetes and control patients $(p<0.05)$.

\section{Discussion}

Malignant tumors and DM are serious public health issues worldwide. This phenomenon is especially prevalent in Hungary, as this country ranks first in oral cancer morbidity and mortality in Europe (19).

Oral squamous cell carcinoma has a poor prognosis, with the five-year survival rate remaining at 50-55\% $(14,20)$. Numerous medical publications have confirmed the correlation between DM and malignant tumors $(10,12,21)$. In particular, head, neck and oral tumors exist more frequently in patients with diabetes. Diabetes mellitus increases the progression of oral carcinomas and reduces life expectancy (14).

Several tissue reactions promote the spread of malignant tumors in DM patients. In type 2 diabetes, the permanent hyperglycemic condition generates the release of free radicals and oxidative stress (22).

The elevated glucose concentration facilitates the synthesis of tumor cell DNA, as malignant cell metabolism relies on the pentose phosphate pathway. Due to oxidative balance failure, advanced glycation end products (AGEs) accumulate, which generates the release of free radicals, cytokines and growth factors (23). These harmful products cause extracellular matrix damage and increase the permeability of the basal membrane, thereby promoting the spread of cancers. It has already been shown that matrix metalloproteinase levels increase in DM, which facilitates the local distribution of tumor cells and the formation of metastases $(17,24)$. AGEs enhance the expression of their receptor, RAGE, which is one of the main regulatory factors underlying tumor cell invasion (25). Hyperglycemia also increases the agglutination of blood cells, which causes microembolization, blood vessel obstruction and hypoxia (26). As non-oxidative anabolic pathways play a leading role in tumor cell metabolism, these cells are able to multiply even in hypoxic conditions (27) In cases of diabetes, elevated glucose levels potentiate ribose generation and DNA replication by tumor cells (28). Hyperglycemia also promotes 
Végh et al: Type-2 Diabetes Mellitus and Oral Tumors in Hungary

Table II. Oral cancer locations among patients with and without diabetes examined between the years 2012 and 2015.

Location of oral cancers

\begin{tabular}{|c|c|c|c|c|c|c|}
\hline & Labial tumor & Lingual tumor & Sublingual tumor & Gingival tumor & Other tumor & Total \\
\hline NON-DM & $21.7 \%(122)$ & $18.5 \%(104)$ & $22.1 \%(124)$ & $30.2 \%(170)$ & $7.5 \%(42)$ & 562 \\
\hline DM & $34.7 \%(68)$ & $21.4 \%(42)$ & $13.3 \%(26)$ & $19.4 \%(38)$ & $11.2 \%(22)$ & 196 \\
\hline Total & $25 \%(190)$ & $19.3 \%(146)$ & $19.9 \%(150)$ & $27.4 \%(208)$ & $8.4 \%(64)$ & 758 \\
\hline
\end{tabular}

DM, Diabetes mellitus.

the spread of carcinomas and tissue damage due to the Glut-1 glucose transporter. Indeed, the rate of Glut-1 expression significantly correlates with the increased mortality and lower life expectancy of oral cancer patients (3).

Our comparative epidemiological study showed that the rate of type 2 diabetes increased in the tumor group from $14.6 \%$ to $25.9 \%$ within 14 years, as did the proportion of patients with impaired glucose tolerance from $9.7 \%$ to $20.6 \%$. Based on these data, $46.5 \%$ of oral cancer patients had a glucose metabolism disorder.

Another remarkable change among oral cancer patients came in the form of gender distribution. The former study showed a predominance of males (435 males, 175 females), while the second study reflected an equalization of the sexes (400 males, 358 females). The average age of the patients also changed compared to the previous study. The average age of diabetes patients with oral cancer increased from 60 to 65 years, likely due to the higher participation of female patients. Malignant tumors of women typically appear after menopause when the estrogenic defensive mechanisms no longer exist; this hormonal change also contributes to obesity and insulin resistance $(2,29)$.

Persistent hyperglycemia and hyperinsulinemia can both provoke pathological cell proliferation. Insulin has several effects on metabolism as insulin itself is a growth factor that facilitates the production of other growth factors (IGFs) and increases the mitogenic activity of these products. Growth factors, such as insulin, regulate the growth, proliferation, differentiation and malignant degeneration of cells $(4,6,30)$.

The most frequent types of cancer in the new study among patients with diabetes were labial and lingual carcinomas, in contrast with the previous study where the most common tumor locations were labial and gingival tumors. The salivary secretion rate of patients with diabetes usually decreases, especially when patients are not attended to adequately. Furthermore, cheilitis and glossitis can also be present, with these forms of chronic inflammation and atrophy serving as precursors to malignancy.

In the latter study, the most frequent locations of cancer among non-diabetes patients were the gingiva and the sublingual, whereas the former study showed lingual and sublingual dominance. The most considerable risk factors for
Table III. Prevalence of smoking among oral cancer, oral cancer and diabetes, as well as control patients.

\begin{tabular}{lcc}
\hline & \multicolumn{2}{c}{ Frequency of smoking } \\
\cline { 2 - 3 } & $1998-2002$ & $2012-2015$ \\
\hline Oral cancer & $68 \%$ & $57.7 \%$ \\
Oral cancer + DM & $30 \%$ & $64.3 \%$ \\
Control & $28 \%$ & $41.2 \%$ \\
\hline
\end{tabular}

DM, Diabetes mellitus.

oral cancer are smoking and excessive alcohol consumption, with these factors manifesting their harmful effects primarily on the sublingual and lingual areas. Carcinogenic compounds in tobacco accumulate in saliva on the sublingual region, which forms a horseshoe-like area between the tongue and the base of the mouth. Hard alcohol consumption increases the permeability of the mucosa and promotes the access of carcinogenic products through the epithelial barrier.

These processes contribute to sublingual carcinomas, which were the most common tumors in non-diabetes patients in the previous study. However, in the more recent examination period, we observed gingival tumors in addition to sublingual cancer as the most frequent tumor types. This difference can be explained by the increased rate of women included in the study, as gingival hormone receptors are sensitive to sex hormone changes (such as in the case of pregnancy gingivitis and epulis). We hypothesize that the increased occurrence of gingival tumors was generated by the hormonal changes that take place after menopause.

The frequency of smoking among oral cancer patients slightly decreased over the previous 14 years from $68 \%$ to $57.7 \%$. In contrast, the rate of smoking among cancer patients, who also had diabetes, significantly increased from $30 \%$ to $64.3 \%$. These data are similar to those from other previous studies, which report a smoking rate of $70 \%$ in the same patient group $(17,31)$. In the control group, the frequency of smoking also increased compared to the data obtained in the earlier study. 
Data provided by the World Health Organization (WHO) showed that the smoking related morbidity in Hungary is approximately two times higher than the average in the European Union (15), a phenomenon likely caused by social and economic conditions. After the economic crisis of 2008, many individuals in Hungary between 50-60 years of age became unemployed and it became more difficult to find work, especially for unqualified individuals, who likely constituted the elderly and smoker group in this study.

Smoking induces free radical reactions and metabolic pathway disorders. Due to these processes, smoking plays a major role in tumor formation and insulin resistance $(32,33)$.

As diabetes is a risk factor for oral complications, annual oral screening needs to be highlighted in diabetes care guidelines (34). We would like to draw attention to the rapidly increasing number of oral cancer patients with glucose metabolism disorders and highlight the importance of regular stomato-oncological screening and interdisciplinary cooperation between medical professionals in the fields of type 2 diabetes and oral cancer.

\section{Acknowledgements}

This research did not receive any specific grant or financial support from funding agencies in the public, commercial or not-for-profit sectors. The Authors have no financial or other interest to declare in relation to the content of this manuscript.

\section{References}

1 International Diabetes Federation. IDF diabetes atlas. 7th ed. Brussels, Belgium: International Diabetes Federation, 2015.

2 Gupta K, Krishnaswamy G, Karnad A and Peiris AN: Insulin: A novel factor in carcinogenesis. Am J Med Sci 323: 140-145, 2002.

3 Kunkel M, Reichert TE, Benz P, Lehr HA, Jeong JH, Wieland $\mathrm{S}$, Bartenstein P, Wagner W and Whiteside TL: Overexpression of Glut-1 and increased glucose metabolism in tumors are associated with a poor prognosis in patients with oral squamous cell carcinoma. Cancer 97: 1015-1024, 2003.

4 Werner $\mathrm{H}$ and Katz $\mathrm{J}$ : The emerging role of the insulin-like growth factors in oral biology. J Dent Res 83: 832-836, 2004.

5 Bhawal UK, Ozaki Y, Nishimura M, Sugiyama M, Sasahira T, Nomura Y, Sato F, Fujimoto K, Sasaki N, Ikeda MA, Tsuji K, Kuniyasu $\mathrm{H}$ and Kato Y: Association of expression of receptor for advanced glycation end products and invasive activity of oral squamous cell carcinoma. Oncology 69: 246-255, 2005.

6 Carboni JM, Lee AV, Hadsell DL, Rowley BR, Lee FY, Bol DK, Camuso AE, Gottardis M, Greer AF, Ho CP, Hurlburt W, Li A, Saulnier M, Velaparthi U, Wang C, Wen ML, Westhouse RA, Wittman M, Zimmermann K, Rupnow BA and Wong TW: Tumor development by transgenic expression of a constitutively active insulin-like growth factor I receptor. Cancer Res 65: 3781-3787, 2005.

7 Jee SH, Ohrr H, Sull JW, Yun JE, Ji M and Samet JM: Fasting serum glucose level and cancer risk in Korean men and women. JAMA 293: 194-202, 2005.
8 Inoue M, Iwasaki M, Otani T, Sasazuki S, Noda M and Tsugane $\mathrm{S}$ : Diabetes mellitus and the risk of cancer: results from a largescale population-based cohort study in Japan. Arch Intern Med 166: 1871-1877, 2006.

9 Saini R, Al-Maweri SA, Saini D, Ismail NM and Ismail AR: Oral mucosal lesions in non oral habit diabetic patients and association of diabetes mellitus with oral precancerous lesions. Diabetes Res Clin Pract 89: 320-326, 2010.

$10 \mathrm{Wu} \mathrm{CH}, \mathrm{Wu}$ TY, Li CC, Lui MT, Chang KW and Kao SY : Impact of diabetes mellitus on the prognosis of patients with oral squamous cell carcinoma: A retrospective cohort study. Ann Surg Oncol 17: 2175-2183, 2010.

11 Esposito K, Chiodini P, Colao A, Lenzi A and Giugliano D: Metabolic syndrome and risk of cancer: A systematic review and meta-analysis. Diabetes Care 35: 2402-2411, 2012.

12 Faulds $\mathrm{MH}$ and Dahlman-Wright K: Metabolic diseases and cancer risk. Curr Opin Oncol 24: 58-61, 2012.

13 Ranc K, Jorgensen ME, Friis S and Carstensen B: Mortality after cancer among patients with diabetes mellitus: Effect of diabetes duration and treatment. Diabetologia 57: 927-934, 2014.

14 Tseng KS, Lin C, Lin YS and Weng SF: Risk of head and neck cancer in patients with diabetes mellitus: A retrospective cohort study in Taiwan. JAMA Otolaryngol Head Neck Surg 140: 746-753, 2014.

15 HFA: European Health for All Database (HFA-DB). WHO Regional Office for Europe, Copenhagen, 2009.

16 Gong Y, Wei B, Yu L and Pan W: Type 2 diabetes mellitus and risk of oral cancer and precancerous lesions: A meta-analysis of observational studies. Oral Oncol 51: 332-340, 2015.

17 Ujpál M, Matos O, Bibok G, Somogyi A, Szabó G and Suba Z: Diabetes and oral tumors in Hungary: Epidemiological correlations. Diabetes Care 27: 770-774, 2004.

18 Ujpál M, Barabás J, Kovalszky I, Szabó G, Németh Z, Gábris K and Suba Z: A preliminary comparative study of the prognostic implications of type 2 diabetes mellitus for patients with primary gingival carcinoma treated with surgery and radiation therapy. $\mathrm{J}$ Oral Maxillofac Surg 65: 452-456, 2007.

19 Dobrossy L: Cancer mortality in central-eastern Europe: Facts behind the figures. Lancet Oncol 3: 374-381, 2002.

20.Neville BW and Day TA: Oral cancer and precancerous lesions. CA Cancer J Clin 52: 195-215, 2002.

21 Goutzanis L, Vairaktaris E, Yapijakis C, Kavantzas N, Nkenke E, Derka S, Vassiliou S, Acil Y, Kessler P, Stavrianeas N, Perrea D, Donta I, Skandalakis P and Patsouris E: Diabetes may increase risk for oral cancer through the insulin receptor substrate-1 and focal adhesion kinase pathway. Oral Oncol 43: 165-173, 2007.

22 Baynes JW and Thorpe SR: Role of oxidative stress in diabetic complications: A new perspective on an old paradigm. Diabetes 48: 1-9, 1999.

23 Salahudeen AK, Kanji V, Reckelhoff JF and Schmidt AM: Pathogenesis of diabetic nephropathy: A radical approach. Nephrol Dial Transplant 12: 664-668, 1997.

24 Chen HH, Chen IH, Liao CT, Wei FC, Lee LY and Huang SF: Preoperative circulating C-reactive protein levels predict pathological aggressiveness in oral squamous cell carcinoma: A retrospective clinical study. Clin Otolaryngol 36: 147-153, 2011.

25 Schmidt AM, Hori O, Cao R, Yan SD, Brett J, Wautier JL, Ogawa S, Kuwabara K, Matsumoto M and Stern D: RAGE: A novel cellular receptor for advanced glycation end products. Diabetes 45: S77-S80, 1996. 
26 Lorenzi M: Glucose toxicity in the vascular complications of diabetes: The cellular perspective. Diabetes Metab Rev 8: 85103, 1992.

27 Brennan PA, Mackenzie N and Quintero M: Hypoxia-inducible factor $1 \alpha$ in oral cancer. J Oral Pathol Med 34: 385-389, 2005.

28 Boros LG, Cascante M and Paul Lee WN: Metabolic profiling of cell growth and death in cancer: Applications in drug discovery. Drug Discov Today 7: 364-372, 2002.

29 Barchiesi F, Jackson EK, Gillespie DG, Zacharia LC, Fingerle J and Dubey RK: Methoxyestradiols mediate estradiol-induced antimitogenesis in human aortic SMCs. Hypertension 39: 874$879,2002$.

30 Suba $\mathrm{Z}$ and Ujpal M: Correlations of insulin resistance and neoplasms. Magy Onkol 50: 127-135, 2006.

31 Albrecht M, Bánóczy J, Dinya E and Tamás G Jr.: Occurrence of oral leukoplakia and lichen planus in diabetes mellitus. J Oral Pathol Med 21: 364-366, 1992.
32 Foy CG, Bell RA, Farmer DF, Goff DC Jr. and Wagenknecht LE: Smoking and incidence of diabetes among U.S. adults: Findings from the insulin resistance atherosclerosis study. Diabetes Care 28: 2501-2507, 2005.

33 Ishizaka N, Ishizaka Y, Toda E, Nagai R and Yamakado M: Association between smoking, hematological parameters, and metabolic syndrome in Japanese men. Diabetes Care 29: 741, 2006.

34 Mayard-Pons ML, Rilliard F, Libersa JC, Musset AM and Farge P: Database analysis of a French type 2 diabetic population shows a specific age pattern of tooth extractions and correlates health care utilization. J Diabetes Complicat 29: 993-997, 2015.

Received January 31, 2017

Revised March 3, 2017

Accepted March 6, 2017 